\title{
Target Tracking for Networked Moving Camera using Unsynchronous Multiple Sensor Data
}

\author{
${ }^{\dagger}$ Tomohiro Umetani, ${ }^{\dagger}$ Masahiro Tanaka, ${ }^{\dagger}$ Masahiro Wada and ${ }^{\ddagger}$ Minoru Ito \\ $\dagger$ Department of Intelligence and Informatics, Konan University \\ 8-9-1 Okamoto, Higashinada, Kobe, Hyogo 658-8501, JAPAN \\ $\ddagger$ Department of Control Engineering, Maizuru National College of Technology \\ 234 Shiroya, Maizuru, Kyoto 625-8511, JAPAN \\ E-mail: umetani@konan-u.ac.jp
}

\begin{abstract}
This paper describes a method for target-tracking by a moving camera such as a mobile robot based on a scheme of the fusion of the unsynchronous sensor data. To realize the interaction between the person and the robot, we need not only the location of the target but the relation of the target and the robot such as the motion plan of the target. This study focuses on the sensor data fusion scheme to estimate the relation between the robot and the targets. We apply a fuzzy inference for the sensor data fusion since the result of the fuzzy inference is useful for the fuzzy controller of the moving camera. In addition, the paper discusses the implementation of the fuzzy inference system for the target tracking as an RT component for a distributed component middleware. Experimental results show the feasibility of the proposed method.
\end{abstract}

\section{Introduction}

The authors have been developing a moving robot, which we call a "campus robot" [1] based on "RTComponent framework" [2]. In the campus robot project, the simultaneously localization and object detection method using mixture PDF model has been developed [3]. For achieving tasks for communication with people and following the target person, the tracking people method is a crucial issue as well as the localization of the mobile robot. In this case, vision systems include cameras are widely used for tracking; the recognition based on vision system has uncertainty. Therefore, the integration of the other sensor data for validation of the recognition result is desirable.

We have proposed the probabilistic model of the data fusion of thermal image for the validation of the recognition result [4]. The frame rate of thermal image is different from that of the vision system. This method uses unsynchronous updating the model of the tracking target by measurement data of the camera and the thermal imaging sensor.

This paper describes a method for target-tracking by a moving camera such as a mobile robot based on a

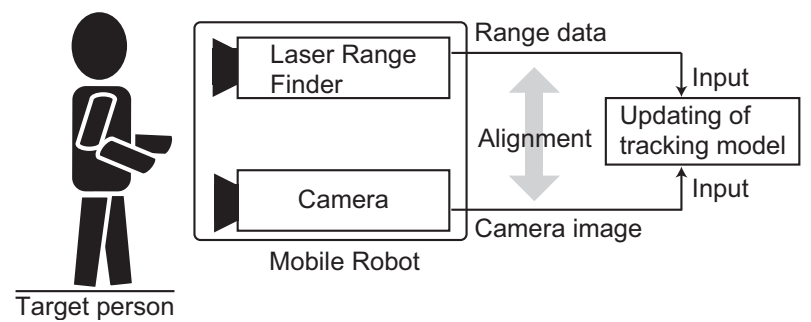

Fig. 1: Basic idea of integration of multiple imaging data for target-tracking.

scheme of the fusion of the unsynchronous sensor data. Figure 1 shows an outline of the integration of multiple imaging data for target-tracking. To realize the interaction between the person and the robot, we need not only the location of the target but the relation of the target and the robot such as the motion plan of the target. In this case, the moving camera needs the series of the tracking results from multiple data acquisition; however, it is difficult to use the estimation results directly for target tracking since the estimation results have uncertainty.

The paper focuses on the sensor data fusion scheme to estimate the relation between the robot and the targets [5]. We apply a fuzzy inference for the sensor data fusion since the result of the fuzzy inference is useful for the fuzzy controller of the moving camera. In addition, we discuss the implementation of the fuzzy inference system for the target tracking as an RT component for a distributed component middleware [2]. Experimental results show the feasibility of the proposed method.

\section{Methodology}

This study deals with a target-tracking problem for a mobile robot. Figure 1 shows an outline of the integration of multiple imaging data for target-tracking. The robot obtains the multiple sensor data unsynchronously. The frame rate of image data is different from that of the other sensor data. Moreover, this problem appears when we apply the multiple image- 
tracking methods for the obtained image data. In addition, due to the delay of the network and issues of time management of the distributed robot system, it is not expectable that the sensor data from each device are obtained synchronously. Therefore, we apply an unsynchronous updating model of the tracking target by measurement data.

We focus on a fuzzy inference system for the sensor data fusion since the result of the fuzzy inference is useful for the fuzzy controller of the moving camera. For example, Amanatiadis proposes an indoor navigation system based on sensor data fusion from inertial data, vision data, and RFID data [6]. The system outputs the quality of the localization results using fuzzy inference technique. The user can switch the localization methods according to the localization results based on various methods.

We apply a fuzzy inference system to evaluate the relation of the target person and the robots in order to control the mobile robot navigation. For the mobile robot, the target tracking problem is regarded as the detection problem of the change of displacement between the robot and the target person. In this paper, we use a fixed generic USB camera and a laser range finder. The number of target person is one. To detect the location of the person, we apply the face detector using the Haar-Like descriptor for the captured image sequences.

\section{$3 \quad$ Fuzzy Inference System}

This section describes a method for the fuzzy inference systems using the multiple sensing data sources. First, the system configuration is explained. Next, we introduce a fuzzy inference system using the image sequences and the range data. Finally, a fuzzy inference system based on the data fusion scheme and experimental results are illustrated.

\subsection{System configuration}

As shown in Fig. 1, we use a Hokuyo URG-04LXUG01 laser range finder and a generic camera device attached to the notebook PC for image sensing. The laser range finder is connected to $\mathrm{PC}$. We develop the experimental system using OpenRTM-aist [7]. The control software of the camera and the laser range finder, the fuzzy inference systems, and the viewer for the tracking results are constructed by the RT components. These RT components are executed unsynchronously in the PC. We use the OpenCV library [8] to implement the Haar-Like face detector for person tracking.

We construct three RT-components shown in Table 1. "C_Image", "O_Image" and "R_Data" mean the camera image, the camera image that the result of detection of the target person is overlapped, and the range data captured by the laser range finder. "C_Result" and "R_Result" mean the results of the fuzzy inference
Table 1: Specs of RT-Components.

\begin{tabular}{c|cc}
\hline Component & Input & Output \\
\hline \hline \multirow{2}{*}{ fuzzCamTrack } & C_Image & O_Image \\
C_Result \\
\hline fuzzLRFTrack & R_Data & R_Result \\
\hline \multirow{2}{*}{ fuzzTrack } & O_Image & \\
& C_Result & O_Image \\
& R_Result & \\
\hline
\end{tabular}

based on the camera image and range data. Component "fuzzCamTrack" receives the camera image, then it outputs the detection result and inference results by the camera image. Component "fuzzLRFTrack" receives the range data from the laser range finder, then it outputs the estimation results by the fuzzy inference system. Component "fuzzTrack" receives the estimation results of the target person from the former components, then the component estimates the status of the target person, and it outputs the overlay image of the detection result. We use three other components for capturing the camera image and the range data, and visualizing the detection results; these components are reused from the other research projects. These six components are executed on the PC independently.

The frame rate of the vision system is different from the frequency of the laser range finder. The frame rate of a generic USB camera is 30 frames per second, on the other hand, the frequency of the data acquisition of the laser range finder is $10 \mathrm{~Hz}$. We apply an asynchronous inference system of the tracking target by the measurement data of the camera and the laser range finder.

\subsection{Detection of motion of target person using image sequences}

The input values of the inference system are the center of the gravity of the face area of the target, and the result of the past period of the output of the inference system. We define the three membership functions according to the moving distance of the position of target person's face. Figure 2 shows the membership functions for the three fuzzy numbers on the moving displacement in the fuzzy inference system.

Next, we construct an inference system based on the fuzzy inference scheme. Table 2 shows the IF-THEN rules for the fuzzy inference system. The output of the system is the status of the target person, "no moving," "possible" and "active." The codes 'ZO,' 'S' and 'L' in Table 2 correspond to "very small," "small," and "large," respectively. The codes 'NM,' 'P' and 'A' correspond to "no moving," "possible," and "active," respectively. The code "*' means any condition. In this scheme, the fuzzy system can use the former output result of the system as the input of the present time; the system outputs the inference results taking account into the former states of the tracking person. There- 


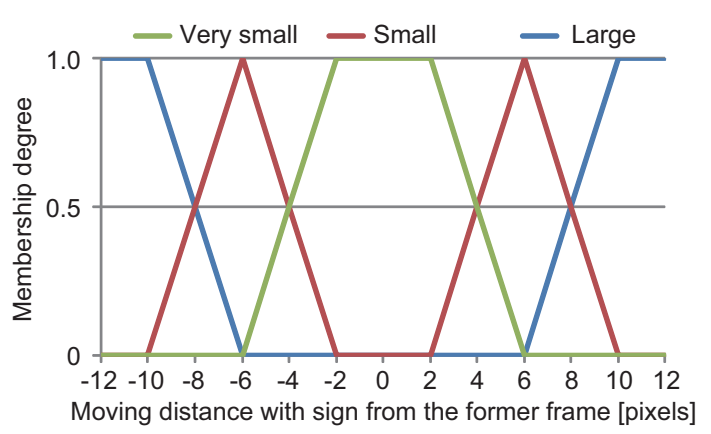

Fig. 2: Membership function of moving distance in camera image for the status of tracking target.

Table 2: IF-THEN rule for fuzzy inference using camera image sequences.

\begin{tabular}{cccc}
\hline Rule & Input & $\begin{array}{c}\text { Output of } \\
\text { former frame }\end{array}$ & Output \\
\hline 1 & ZO & N, P & NM \\
2 & $\mathrm{~S}$ & N, P & P \\
3 & L & $*$ & $\mathrm{~A}$ \\
4 & ZO & A & $\mathrm{P}$ \\
5 & $\mathrm{~S}$ & $\mathrm{~A}$ & $\mathrm{P}$ \\
\hline
\end{tabular}

fore, the smoothed output results are expected; these properties are useful for elements of the control input for the mobile robots.

We have conducted the tracking and estimation of the moving conditions using the fuzzy inference system. We detect and track the target person with in 50 frames of the images. Since the calculation cost for face detection process is high, the frame rate of the detection is 56 [frame/s]. The conditions of the target person are set (1) the person does not move in the measurement interval, (2) the person stops at the beginning of the interval and moves to left or right side of the image frame, then the person stops at the end of the interval, and (3) the person moves to left and right side of the image frame all the interval. We set the conditions that the target person moves more slowly for the conditions (2) and (3). The number of the experimental conditions is five.

Tables 3-5 show the estimation results for the states of the target person under the conditions that the person does not move in the interval, the person moves to left and right side of the image frame, and the person moves slowly in the image frame. From the experimental results, the estimation system outputs the "possible" signals on when the target person moves slightly. When the target person moves in the image frame, the target person stops in the frame immediately on the switching the moving direction; the estimation system outputs the "possible" signals according to the outputs of the former frames. When the target person moves slowly in the image frame, the estimation system out-
Table 3: Experimental result (motionless).

\begin{tabular}{cc}
\hline Estimation result & Frames \\
\hline Motionless & 48 \\
Possible for moving & 2 \\
Moving & 0 \\
\hline
\end{tabular}

Table 4: Experimental result (moving).

\begin{tabular}{cc}
\hline Estimation result & Frames \\
\hline Motionless & 0 \\
Possible for moving & 17 \\
Moving & 33 \\
\hline
\end{tabular}

Table 5: Experimental result (moving slowly).

\begin{tabular}{cc}
\hline Estimation result & Frames \\
\hline Motionless & 10 \\
Possible for moving & 31 \\
Moving & 9 \\
\hline
\end{tabular}

puts the "no moving" and "possible" signals.

\subsection{Detection of motion of target person using range data}

The input values of the inference system are the center of the gravity of the range data of the target person and the change of displacement of the target person. We obtain the displacement of the target person using the range data as following procedure:

1. The RT component for fuzzy inference receives the range data of surroundings.

2. The component removes the range data corresponding to the wall, shelves, and so on. In this experiment, the range data that is within the $\pm 1.8[\mathrm{~m}]$ of width, and $5[\mathrm{~m}]$ of the distance from the laser range finder are used for the calculation of the target person.

3. The position and distance of the target position are estimated by the filtered range data. The displacement and the change of the displacement from that of the former data acquisition are calculated.

Figures 3 and 4 show the membership functions for the fuzzy inference system for the displacement of the target person from the laser range finder and the change of displacement per $1[\mathrm{~s}]$ of the target person. Then, we define the fuzzy rules "near," "middle" and "far" for the displacement of the target person, and "close," "stay," "step away" for the change of displacement per $1[\mathrm{~s}]$ of the person. 


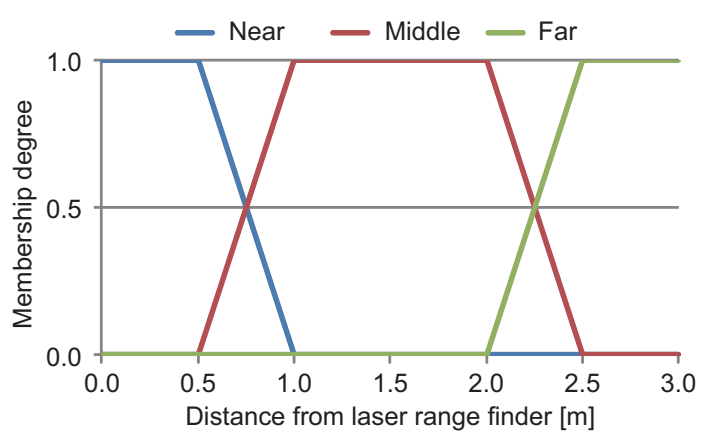

Fig. 3: Membership function of distance of range data for the status of tracking target.

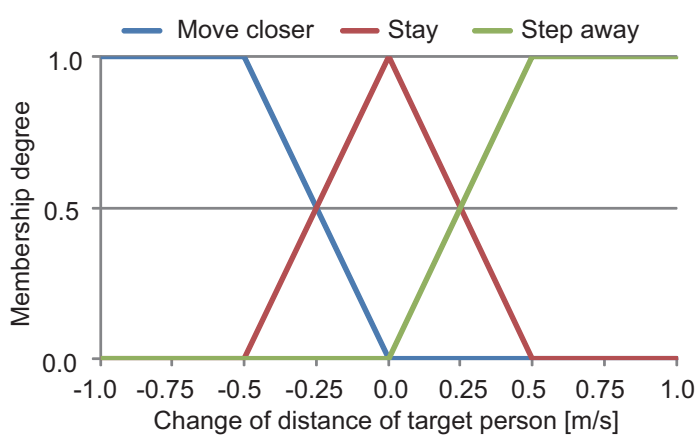

Fig. 4: Membership function of change of distance of range data for the status of tracking target.

We construct an inference system based on the fuzzy inference scheme. Table 6 shows the IF-THEN rules for the fuzzy inference system using range data. The output of the system is the status of the target person, "danger (D)," "caution (CA)" and "safe (SA)." The codes 'N,' 'M' and 'F' in Table 2 correspond to "near," "middle," and "far," respectively. The codes 'C,' 'ST' and 'AW' correspond to "moving closer," "stay," and "step away," respectively.

\subsection{Fuzzy inference system using data fusion}

We apply the fuzzy inference system using the values of the membership functions and the results of the output of the fuzzy inference system using the camera images and the range data. We use three other components for capturing the camera image and the range data, and visualizing the detection results; these components are reused from the robot software modules developed in the other research projects $[9,10]$. The RT components for the experiment are connected shown as Fig. 5, then the total person tracking system can be executed. Component "fuzzCamTrak" and "fuzzLRFTrack" send the values of the membership functions as the tracking results of each sensor data. Component "fuzzTrack" receives the values of the membership functions unsynchronously; the component makes the fuzzy inference using the received data. If the component cannot use the updated values of the membership functions from the former components, the component
Table 6: IF-THEN rule for fuzzy inference using range data.

\begin{tabular}{cccc}
\hline Rule & Displacement & $\begin{array}{c}\text { Change of } \\
\text { Distance }\end{array}$ & Output \\
\hline 1 & $\mathrm{~N}$ & $\mathrm{C}, \mathrm{ST}$ & $\mathrm{D}$ \\
2 & $\mathrm{~N}$ & $\mathrm{AW}$ & $\mathrm{CA}$ \\
3 & $\mathrm{M}$ & $\mathrm{C}$ & $\mathrm{D}$ \\
4 & $\mathrm{M}$ & $\mathrm{ST}$ & $\mathrm{CA}$ \\
5 & $\mathrm{M}$ & $\mathrm{AW}$ & $\mathrm{SA}$ \\
6 & $\mathrm{~F}$ & $\mathrm{C}$ & $\mathrm{CA}$ \\
7 & $\mathrm{~F}$ & $\mathrm{ST}, \mathrm{AW}$ & $\mathrm{SA}$ \\
\hline
\end{tabular}

makes the inference using the values that are received in the former frames.

In this experiment, we estimate the "safety" of the mobile robot using the movement, displacement and the change of displacemnet of the target person. Three membership functions $\mu_{s}$ (safe), $\mu_{c}$ (caution) and $\mu_{d}$ (danger) are defined as follows:

$$
\begin{aligned}
\mu_{s}= & \left(\mu_{Z O} \wedge \mu_{F} \wedge\left(\mu_{S T} \vee \mu_{A W}^{\frac{1}{2}}\right)\right) \\
& \vee\left(\mu_{Z O} \wedge \mu_{M} \wedge \mu_{A W}^{2}\right) \\
& \vee\left(\left(\mu_{S} \vee \mu_{L}\right) \wedge \mu_{F} \wedge \mu_{A W}\right) \\
& \vee\left(\mu_{S} \wedge \mu_{M} \wedge \mu_{A W}^{2}\right) \vee\left(\mu_{F} \wedge \mu_{S T}\right) \\
\mu_{c}= & \left(\mu_{N} \wedge \mu_{A W}^{2}\right) \\
& \vee\left(\mu_{M} \wedge\left(\mu_{S} \vee \mu_{L}\right) \wedge\left(\mu_{S T} \vee \mu_{A W}\right)\right) \\
& \vee\left(\mu_{F} \wedge \mu_{C}\right) \\
& \left(\left(\mu_{N} \vee \mu_{M}\right) \wedge \mu_{C} \wedge \neg \mu_{A W}\right) \\
& \vee\left(\mu_{C} \wedge \mu_{S T} \wedge \neg\left(\mu_{M} \vee \mu_{F}\right)\right)
\end{aligned}
$$

where $\mu_{Z O}, \mu_{S}, \mu_{L}, \mu_{C}, \mu_{S T}, \mu_{A W}, \mu_{N}, \mu_{M}$ and $\mu_{F}$ indicate the membership degree of the functions "very small," "small," "large," "moving closer," "stay," "step away," "near," "middle," and "far," respectively. The output of the system is the status of the target person, "safe," "caution" and "danger" as the degree of the membership functions.

We implement the fuzzy inference system as the RT components that execute independently and unsynchronously. The system outputs the safety of the robot when the image data or the range data are obtained. To control the mobile robot, the fuzzy controller for the velocity of the mobile robots using the fuzzy number $\mu_{s}, \mu_{c}$ and $\mu_{d}$ are needed. The control problem of the mobile robot is one of the feature works.

From the experimental results, the estimation system outputs composed fuzzy inference results. In this experiment, the experimental conditions and fuzzy rules are fundamental. The target detection and measurement based on the estimation of the reflection targets of the laser scan using range data [11] is one of expansion of this study. Construction of fuzzy controller for mobile robots using the fuzzy inference schema, the 


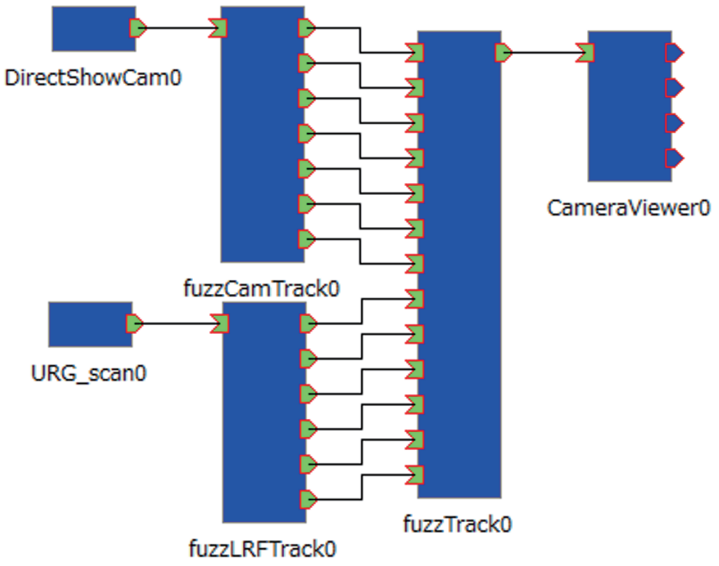

Fig. 5: Connection of RT components for experiment.

target tracking for the communication tasks with the person will be addressed as future works.

\section{Conclusion}

This paper describes a method for target-tracking by a moving camera such as a mobile robot based on a scheme of the fusion of the unsynchronous sensor data. In this study, we focus on the sensor data fusion scheme to estimate the relation between the robot and the targets. We apply a fuzzy inference for the sensor data fusion since the result of the fuzzy inference is useful for the fuzzy controller of the moving camera. In addition, we discuss the implementation of the fuzzy inference system for the target tracking as an RT component for a distributed component middleware.

Multiple target-person tracking using sensor data fusion, construction of fuzzy controller for mobile robots using the fuzzy inference schema, the target tracking for the communication tasks with the person will be addressed as future works.

\section{Acknowledgment}

This work was supported in part by the JSPS Grantin-Aid for Scientific Research \#24500288, and Ministry of Education, Culure, Sports, Science and Technology in Japan.

\section{References}

[1] M. Wada, M. Tanaka, T. Umetani and M. Ito, "Framework of Control and Stabilization for an Inverted Pendulum Moving Robot," in: Proc. 42nd ISCIE International Symposium on Stochastic Systems Theory and Its Application, pp. 1-5, 2011.

[2] N. Ando, T. Suehiro, K. Kitagaki, T. Kotoku and W. K. Yoon, "RT-Middleware: Distributed Com- ponent Middleware for RT (Robot Technology)," in: Proc. 2005 IEEE/RSJ International Conference on Intelligent Robots and Systems, pp. 35553560, 2005 .

[3] M. Tanaka, M. Wada, T. Umetani and M. Ito, "Detection of Mobile Objects by Mixture PDF Model for Mobile Robots," Transactions of the Institute of Systems, Control and Information Engineers, vol. 25, no. 11, pp. 308-315, 2012.

[4] T. Umetani, N. Kuga, M. Tanaka, M. Wada and M. Ito, "Target-Tracking based on Fusion of Unsynchronized Sensor Data from Vision System and Thermal Imaging Sensor," Transactions of the Institute of Systems, Control and Information Engineers, vol. 25, no. 11, pp. 323-327, 2012.

[5] T. Umetani, R. Usui, M. Tanaka, M. Wada and M. Ito, "Target Tracking by Mobile Camera based on Unsynchronized Sensor Data Fusion," in: Proc. 57th Annual Conf. ISCIE (in Japanese), 2 pages, 2013.

[6] A. Amanatiadis, A. Gasteratos and D. Koulouriotis, "An intelligent multi-sensor system for first responder indoor navigation," Measurement Science and Technology, vol. 22, issue 11, article no. $114025,2011$.

[7] OpenRTM-aist, http://www.openrtm.org/ (accessed October 22, 2013).

[8] The OpenCV Library, http://sourceforge.net/projects/opencvlibrary/ (accessed October 22, 2013).

[9] S. Hirai, "Robot Software Modules and Their Applications," Journal of the Robotics Society of Japan (in Japanese), vol. 31, no. 1, pp. 43-44, 2013.

[10] M. Yoshimoto, R. Nishi and E. Ueda, "RTComponent for generating the environmental map using MRPT," Proc. 13rd Annual Conf. SICE SI Division (in Japanese), pp. 533-534, 2012.

[11] M. Tanaka, M. Wada, T. Umetani and M. Ito, "Parameter Estimation of Mixture PDF Model for Mobile Robots by EM Algorithm," Abst. 45th ISCIE International Symposium on Stochastic Systems Theory and Its Application, 2013. 